\title{
IMC Based PID Controllers Retuning and Its Performance Assessment
}

\author{
Prasanth Sai Duvvapu a, Dr. A S Rathore ${ }^{\text {b }}$ \\ ${ }^{a}$ Research Scholar, Dept. of Electrical \& Electronics Engineering, \\ Sri Satya Sai University of Technology \& Medical Sciences, Sehore, Bhopal Indore Road, Madhya Pradesh, India \\ b Research Guide, Dept. of Electrical \& Electronics Engineering, \\ Sri Satya Sai University of Technology \& Medical Sciences, Sehore, Bhopal Indore Road, Madhya Pradesh, India
}

Article History: Received: 11 January 2021; Accepted: 27 February 2021; Published online: 5 April 2021

\begin{abstract}
Internal Model Control (IMC) is the basis of a systematic program Q on the parameters of the concept and is based on the many modern control technology control system design. The present research work presents a novel control scheme for tuning PID controllers using Internal Model control with the filter time constant optimized using Bee colony Optimization technique. The robustness is measured by a two-block structured singular value, and the disturbance rejection is measured by the minimum singular value of the integral gain matrix. PID controllers are used widely in Industrial Processes. Criteria based on disturbance rejection and system robustness are proposed to assess the performance of PID controllers. Tuning of PID controllers is accomplished using Internal Model control scheme. In this paper, we propose an optimal filter design IMC IMCPID controller for unstable process better set point tracking. IMC includes tuning of filter constant $\lambda$. The controller is suitable for different values of the filter tuning parameters to achieve the desired response in due IMC method is based on pole-zero offset, including IMC design principle method causes the response sets a good point. However, in a stable long lag time of load disturbances leading to undesirable process which is the result of the IMC control industry. Therefore, IMC has been a popular design process processing industry, especially as a means for adjusting the single-loop, PID controllers.
\end{abstract}

Keywords - Internal Model Control, PID controller, Bee colony optimization, Filter constant, Q-parameters.

\section{Introduction}

During the past decades, the process control techniques have made great advance. Numerous control methods like adaptive control, neural control, fuzzy logic control, ANFIS control are developed. Still, PID controllers are considered as the workhorse of almost all the industrial process control applications due to their structural simplicity and robust performance in a wide range of operating condition. PID controller mainly depends on tuning of the gains the proportional gain, integral time and the derivative time. Tuning the PID controller gains play a major role in deciding the performance. Literature provides many tuning methodologies. IMC is a commonly used technique, which provides a transparent mode for various types of control design and tuning. The proportional-integral (PI), in order to meet the target of most of the control capacity and proportional integral - derivative (PID) controllers, leading to its widespread acceptance in the control industry. Internal Model Control (IMC) based approach to the design of the controller is using the IMC, which is equivalent to the use of one of the IMC PID control in industrial applications. IMC allows a transparent controller design procedure where control quality and robustness are influenced in a direct manner. The IMC concept was conceptualized by approximating the feedback transfer function by Maclaurin's series.the PID controller usually requires some a prior manual retuning to make a Successful industrial application. To bypass this obstacle, an adaptive PID (APID) controller is proposed in this paper which is composed of a PID controller and a fuzzy compensator. Without needing introductory offline learning, the PID controller can automatically online tune the control gains located on the gradient descent method and the fuzzy compensator is designed to eliminate the effect of the approximation errors introduced by the PID controller upon the system stability in the Lyapunov sense. PID controllers are widely used as the preferred controller approach due to their design simplicity and its reliable operation. TheTuning approaches can be detached into two groups which are the conventional and the alternative approaches. Time delay degrades the performance of control system in many industrial application. That too when the time delay is very large, PID controller degrades. The conventional outlooks include the observed methods and the methodical methods which widely used by control designers. The possible approaches are restricted to methods that employ the stochastic process in the tuning rules.Design IMC design process is quite extensive and diverse. Has developed a number of forms; These include single-input, single-output (SISO) and multiple-input, multiple-output (MIMO) formulations, continuous-time and discrete-time design process, the design process unstable open-loop system, combined with feedback - feedforward IMC design and so on. In addition to designing the controller, IMC evaluation and feedback control is related to the basic requirements, such as determining the non-minimum phase element (delay and RHP (RHP) zero) is helpful to realize the impact on control performance. Due to the complexity of the IMC controller depends on the order model and control performance requirements, IMC design also helps determine when simple feedback control structure (such as a PID controller) is sufficient. IMC is a commonly used technique, which provides a transparent mode for various types of control design and tuning. The proportional-integral (PI), in order to meet the target of most of the control capacity and proportional - integral - derivative (PID) controllers, leading to its widespread acceptance in the control industry. Internal Model Control (IMC) based approach to the design of the controller 
is using the IMC, which is equivalent to the use of one of the IMC PID control in industrial applications. Used in industrial process control applications and IMC IMCbased PID controller, there is an optimal filter structure for each specific process model to get the best performance of PID. For a given filter structure, when $\lambda$ decreases, the contradiction between the ideal and the increase in PID controller, while the nominal IMC performance improvement. IMC Background in process control, model-based control system is mainly used to obtain the desired set point and reject small external interference. Internal Model Control (IMC) design is based on the control system contains the control, then you can achieve a perfect control process the fact that some of the statements.

\section{II . LITERATURE REVIEW}

D.Prasanth Sai , et.al [1] Internal Model Control (IMC) is the basis of a systematic program Q on the parameters of the concept and is based on the many modern control technology control system design. What makes IMC particularly attractive is that it presents a method to design the Q-parameterization controller has two basic demands of reality. Therefore, IMC has been a popular design process processing industry, especially as a means for adjusting the single-loop, PID controllers. In this paper, we propose an optimal filter design IMC IMC-PID controller for unstable process better set point tracking. The controller is suitable for different values of the filter tuning parameters to achieve the desired response in due IMC method is based on pole-zero offset, including IMC design principle method causes the response sets a good point. However, in a stable long lag time of load disturbances leading to undesirable process which is the result of the IMC control industry.

U. Sabura Banu, et.al [2] represents the paper as during the past decades, the process control techniques have made great advance. Numerous control methods like adaptive control, neural control, fuzzy logic control, ANFIS control are developed. Still, PID controllers are considered as the workhorse of almost all the industrial process control applications due to their structural simplicity and robust performance in a wide range of operating condition. PID controller mainly depends on tuning of the gains the proportional gain, integral time and the derivative time. Tuning the PID controller gains play a major role in deciding the performance. Literature provides many tuning methodologies. Tuning of PID parameters are based on the exact form of the process expressed by a transfer function. Manufacturers and vendors use different tuning algorithms for designing of the PID control parameters.

\section{IMC TUNING RULE FOR PID CONTROLLERS}

The PID controller based on the IMC tuning rule usually leads to a control loop with a good balance among setpoint tracking, disturbance rejection and robustness, and has been widely adopted in industrial practice for years. From fig 1.2.1 representing the block diagram, the IMC tuning rule for PID controllers is briefly reviewed. Fig. 1. A SISO feedback control loop Consider a SISO feedback control loop depicted in Fig. 1. Here $\mathrm{P}(\mathrm{s})$ and $\mathrm{C}(\mathrm{s})$ are the process and the PID controller, respectively; $\mathrm{r}(\mathrm{t}), \mathrm{u}(\mathrm{t})$ and $\mathrm{y}(\mathrm{t})$ are the setpoint, the control signal, and the process output, respectively. In this context, $\mathrm{P}(\mathrm{s})$ is confined to be a process that is stable, without integrals and negative zeros; hence, the process can be approximated by a first-order plus dead time (FOPDT) model, $\mathrm{P}(\mathrm{s})=\mathrm{K}$ T1 $\mathrm{s}+1 \mathrm{e}-\theta \mathrm{s},(1)$ or a second-order plus dead time (SOPDT) model, $\mathrm{P}(\mathrm{s})=\mathrm{K} \mathrm{T} 1 \mathrm{~s} 2+\mathrm{T} 2 \mathrm{~s}+1 \mathrm{e}$ $-\theta \mathrm{s}$. (2) As $\theta$ is crucial to the subsequent performance index, it is worthy to mention that $\theta$ is the time delay of the low-order model (1) or (2), instead of the time delay of the actual process. For instance, the positive zero of a process can be removed by lumping it into the time delay part of a low-order approximated model .

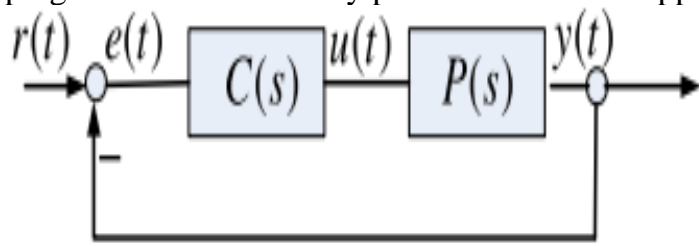

Fig 1.2.1 . Block Diagram Of Imc Tuning

The PID controller C(s) takes a non-interactive formulation, $\mathrm{C}(\mathrm{s})=\mathrm{Kp} 1+1 \mathrm{Tis}+\mathrm{Tds}$. (3) The IMC tuning rule gives the controller setting for the FOPDT model as $\mathrm{Kp}=\mathrm{T} 1 \mathrm{~K}(\tau \mathrm{c}+\theta), \mathrm{Ti}=\mathrm{T} 1, \mathrm{Td}=0$, and that for the SOPDT model as $\mathrm{Kp}=\mathrm{T} 2 \mathrm{~K}(\tau \mathrm{c}+\theta), \mathrm{Ti}=\mathrm{T} 2, \mathrm{Td}=\mathrm{T} 1 \mathrm{~T} 2$. (4) By taking the IMC tuning rule, the desired closed-loop response will be GCL(s) $=1(\theta+\tau \mathrm{c}) \mathrm{s}+\mathrm{e}-\theta \mathrm{s}$ e $-\theta \mathrm{s} \approx 1 \tau \mathrm{cs}+1 \mathrm{e}-\theta \mathrm{s}$. (5) Here the user-selected parameter $\tau \mathrm{c}$ stands for the desired closed-loop time constant. Internal Stabilizer (IS) is a key requirement of any control system theory. In a stable system of internal controls, bounded presented the results of the control system in a bounded output signal everywhere from anywhere in the control system of the input signal. For IMC structure, we have important results of the internal stability of the following:

1. Assuming a perfect internal model $\left(\mathrm{P}=\mathrm{P}^{\wedge}\right)$. IMC control system is internally stable if and only if $\mathrm{p}$ and $\mathrm{q}$ are stable. 


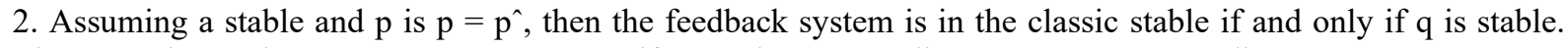
These results apply to IMC structure, even if $\mathrm{p}^{\wedge}$ and $\mathrm{q}$ are nonlinear operator. According to fabless does not match the openloop, linear systems under stable conditions.

\subsection{Performance Assessment of Closed-loop Systems}

It is well-known that a well-designed control system should meet the following requirements besides nominal stability • Disturbance attenuation • Setpoint tracking • Robust stability and/or robust performance The first two requirements are traditionally referred to as 'performance' and the third, 'robustness' of a control system. For robust stability, a common choice of representing uncertainty for a multivariable system is the multiplicative perturbation, and the maximum singular value of the complementary sensitivity matrix is a measure of robustness against this kind of uncertainty, which is usually frequency dependent, and suited for the unmodeled dynamics instead of parameter variations.

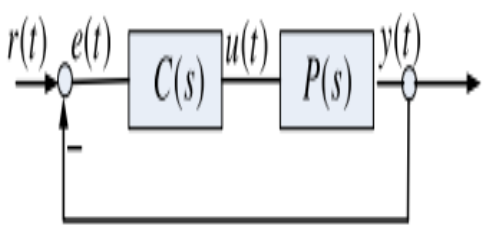

Fig 3.2.1 . Closed-loop Systems

The above figure represents the closed loop system where a simple PID structure consists of three terms which are $K p, K i$ and $K d$ referring to Proportional ,integration and derivative gains respectively. The parallel design of PID controller (after this refers as PID controller)sums the all the error signal, e(t) after being multiplied by PID gains, $\mathrm{Kp}, \mathrm{Ki}$ and $\mathrm{Kd}$ to produce the input signal, u(t). The adjustment process of the values $K p, K i$ and $K d$ is called_tuning' or _design' of PID controller. Criteria based on disturbance rejection and system robustness were proposed to assess the performance of PID controllers.

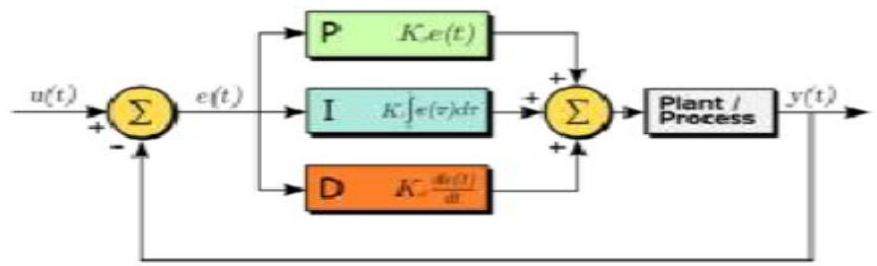

Fig 3.2.2Performance Assessment of Closed-loop Systems

The robustness is measured by a two-block structured singular value, and the disturbance rejection is measured by the minimum singular value of the integral gain matrix. Examples showed that the criteria can be applied to a variety of processes, whether they are stable, integrating or unstable; single-loop or multi-loop. TheTuning approaches can be detached into two groups which are the conventional and the alternative approaches. The conventional outlooks include the observed methods and the methodical methods which widely used by control designers. The possible approaches are restricted to methods that employ the stochastic process in the tuning rules .Stochastic process mentions to one whose behaviour is non-deterministic, where any of its sub-system resolute by the process of deterministic action and a random behaviour. The PID control strategy is named after its three modifying terms, whose sum integrates the manipulated variable (MV). From the fig 1.2.3 Performance Assessment of Closed-loop Systems, the proportional, integral, and derivative terms are computed to calculate the output of the PID controller. Representing $\mathrm{u}(\mathrm{t})$ as the controller output, the final form of the PID algorithm is:

$$
\begin{aligned}
& \mathrm{u}(t)=\mathrm{MV}(t)=K_{p} e(t)+K_{i} \int_{0} e(\tau) d \tau+K_{d} \frac{d}{d t} \mathrm{e}(t) \\
& \text { Where } \\
& \boldsymbol{K}_{p} \text { : Proportional gain, a tuning parameter } \\
& \boldsymbol{K}_{i: \text { Integral gain, a tuning parameter }} \\
& K_{d} \text { : Derivative gain, a tuning parameter } \\
& e: \text { :rror }=S P-P V \\
& t: \text { Time or instantaneous time (the present) } \\
& \mathrm{c}=\text { Variable of integration; takes on values from time } 0 \text { tc } \\
& \text { the present time t. }
\end{aligned}
$$

Where, Proportional gain, a tuning parameter: Integral gain, a tuning parameter: Derivative gain, a tuning parameter: Error: Time or instantaneous time (the present), $\vec{G}=$ Variable of integration; takes on values from time 0 to the present time t. Various combinations of P, I, \& D can be used according to the requirement is various tuning strategies based on an open-loop step response. While they all follow the same basic idea from the above table 1 of the control actions, they differ in slightly in how they extract the model parameters from the recorded response, and also fluctuate marginally as to relate appropriate tuning constants to the model 
parameters. There are four different methods, the classic Ziegler-Nichols open loop test, the Cohen-Coon test, Internal Model Control (IMC) and Approximate M-constrained Integral Gain Optimization (AMIGO). Naturally ifthe response is not sigmoidal or _S' shaped and exhibitsovershoot, or an integrator, then this tuning method is not applicable. Internal Model Controller involves a model based procedure, where the process model is embedded in the controller.

\begin{tabular}{|c|l|l|}
\hline $\begin{array}{c}\text { Control } \\
\text { Actions }\end{array}$ & Estimates & \multicolumn{1}{|c|}{ When to use } \\
\hline P & Present & $\begin{array}{l}\text { Slow response systems, offset } \\
\text { tolerant systems }\end{array}$ \\
\hline I & Past & Too slow to be used often \\
\hline D & Future & $\begin{array}{l}\text { Not used alone because of high } \\
\text { sensitivity and no set point }\end{array}$ \\
\hline PI & $\begin{array}{l}\text { Present \& } \\
\text { Future }\end{array}$ & $\begin{array}{l}\text { Most widely used } \\
\text { PlD }\end{array}$ \\
\hline
\end{tabular}

Table 1 . Control actions

IMC involves a single tunable parameter the filter constant. Consider a linear transfer function model of the process. Figure 1 shows the block diagram of the IMC structure. Internal Model controller involves a model based procedure, where the process model is embedded in the controller. IMC involves a single tunable parameter the filter constant. Consider a linear transfer function model of the process.

\subsection{Internal Model Controller Based PID Design}

Internal Model Controller involves a model based procedure, where the process model is embedded in the controller. IMC involves a single tunable parameter the filter constant. Consider a linear transfer function model of the process. Figure 1 shows the block diagram of the IMC structure. Internal Model controller involves a model based procedure, where the process model is embedded in the controller. IMC involves a single tunable parameter the filter constant. The time delay estimation has been an interesting research topic for years in various areas; see a recent survey in [9]. However, most of the existing methods have their own limitations; in particular, extra experiments are usually required to introduce special signals such as white noise to excite the unknown process. By contrast, it is desired for industrial practice to estimate $\theta$ based on the closed-loop step, ramp or some other simple response, without introducing extra experiments.

- The appearance of $\tau \mathrm{c}$ makes $\eta I A E$ a user specified benchmark, which is indeed an advantage comparing to the MVC benchmark. In other words, users can determine $\tau c$ as the desired closed-loop time constant, and evaluate the performance of the current control loop against the desired one. However, an improper selection of $\tau c$ could make $\eta \mathrm{IAE}$ too large or too small, leading to erroneous conclusion on the control-loop performance. A fair selection of $\tau \mathrm{c}$ is to take the current closed-loop time constant (to be estimated based on the collected data) as $\tau \mathrm{c}$. If $\eta \mathrm{IAE} \rightarrow 1$, the current control-loop performance is close to the expected by using the IMC-tuning rule. Note that $\eta \mathrm{IAE}=1$ is achievable in practice, while the MVC based index for PID control loops is usually quite far away from 1 even though the control-loop performance is satisfactory. Another practical issue is about the noise effect on $\eta I A E$. First, the noise affects the estimates of $\tau c$ and $\theta$, whose accuracies are up to the open- and closedloop identification techniques. Thus, the quality control of the two estimates are out of context here. Second, the noise affects also the calculation of the actual IAE, and may result in an incorrect estimate of $\eta \mathrm{IAE}$, despite a fact that the summation in (14) may enable $\eta \mathrm{IAE}$ somehow robust to noise. To resolve this issue, the noise-free closed-loop response $\mathrm{y}^{\wedge}(\mathrm{t})$ is obtained, i.e., $\mathrm{Y}^{\wedge}(\mathrm{s})=\mathrm{P}^{\wedge}(\mathrm{s}) \mathrm{C}(\mathrm{s}) / 1+\mathrm{P}^{\wedge}(\mathrm{s}) \mathrm{C}(\mathrm{s}) \mathrm{R}(\mathrm{s})$. Based on $\mathrm{y}^{\wedge}(\mathrm{t})$ and $\mathrm{r}(\mathrm{t})$, a noise-free estimate of $\eta \mathrm{IAE}$ can be calculated.

\subsection{IMC STRATEGY}

The process output, $y(s)$, is compared with the output of the model resulting in the signal $d *(s)$. The manipulated input $\mathrm{u}(\mathrm{s})$ is introduced to both the process and its model. Hence the feedback signal send to the controller is $d^{*}(s)=[G p(s)-G p *(s)] \cdot u(s)+d(s)$. 


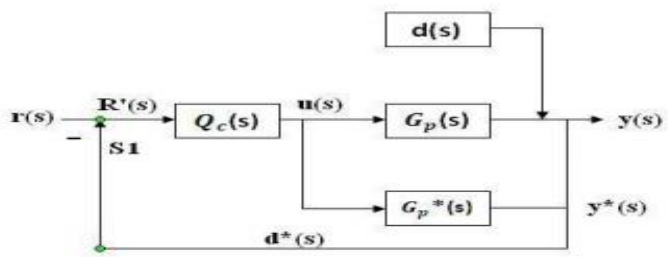

Fig 3.4.1 IMC STRATEGY

In the above figure $3.1, \mathrm{~d}(\mathrm{~s})$ is the unknown disturbance affecting the system. The error signal $\mathrm{r}$ '(s) comprises of the model mismatch and the disturbances which is send as modified set-point to the controller and is given by $r^{\prime}(s)=r(s)-d^{*}(s)$ And output of the controller is the manipulated variable $u(s)$ which is send to both the process and its model. u $(\mathrm{s})=\mathrm{r}^{\prime}(\mathrm{s}) * \mathrm{Qc}(\mathrm{s})=\left[\mathrm{r}(\mathrm{s})-\mathrm{d}^{*}(\mathrm{~s})\right] \mathrm{Qc}(\mathrm{s})=[\mathrm{r}(\mathrm{s})-\{[\mathrm{Gp}(\mathrm{s})-\mathrm{Gp} *(\mathrm{~s})] \cdot \mathrm{u}(\mathrm{s})+\mathrm{d}(\mathrm{s})\}] \cdot \mathrm{Qc}(\mathrm{s}) \mathrm{u}(\mathrm{s})=$ $\left[[\mathrm{r}(\mathrm{s})-\mathrm{d}(\mathrm{s})]^{*} \mathrm{Qc}(\mathrm{s})\right] /[1+\{\mathrm{Gp}(\mathrm{s})-\mathrm{Gp} *(\mathrm{~s})\} \mathrm{Qc}(\mathrm{s})]$ But $\mathrm{y}(\mathrm{s})=\mathrm{Gp}(\mathrm{s}) * \mathrm{u}(\mathrm{s})+\mathrm{d}(\mathrm{s})$ Hence, closed loop transfer function for IMC is $\mathrm{y}(\mathrm{s})=\left\{\mathrm{Qc}(\mathrm{s}) \cdot \mathrm{Gp}(\mathrm{s}) \cdot \mathrm{r}(\mathrm{s})+\left[1-\mathrm{Qc}(\mathrm{s}) \cdot \mathrm{Gp}^{*}(\mathrm{~s})\right] \cdot \mathrm{d}(\mathrm{s})\right\} /\{1+[\mathrm{Gp}(\mathrm{s})-\mathrm{Gp} *(\mathrm{~s})] \mathrm{Qc}(\mathrm{s})\}$ Also improve the system model mismatch effects should be minimized robustness. Since mismatch between the model and the actual process usually occurs in the high frequency response of the system frequency, the low pass filter $\mathrm{F}(\mathrm{s})$ is added to prevent mismatch of. Therefore, the internal model controller is designed to process model, which in series with a low pass filter, i.e. the inverse $\mathrm{Q}(\mathrm{s})=\mathrm{Qc}(\mathrm{s}) * \mathrm{f}(\mathrm{s})$ Order of the filter is selected to be suitable to correct or at least half (e.g., the order is equal to the molecular order of the denominator). The resulting closed loop becomes $y(s)=\left\{Q(s) \cdot G p(s) \cdot r(s)+\left[1-Q(s) \cdot G p^{*}(s)\right] \cdot d(s)\right\} /\left\{1+\left[G p(s)-G p^{*}(s)\right]\right.$ $\mathrm{Q}(\mathrm{s})\}$.

\subsection{SYSTEM DESIGN ANDIMPLEMENTATION}

The IMC-Based PID Control Design Procedure The following steps are used in the IMC-based PID control system design 1. Find the IMC controller transfer function, $q(s)$, which includes a filter, $f(s)$, to make $q(s)$ semiproper or to give it derivative action (order of the numerator of $\mathrm{q}(\mathrm{s})$ is one order greater that the denominator of $\mathrm{q}(\mathrm{s})$ ). Notice that this is a major difference from the IMC procedure. Here, in the IMC-based procedure, we may allow q(s) to be improper, in order to find an equivalent PID controller. The bad news is - you must know the answer that you are looking for, before you can decide whether to make $q(s)$ proper or improper in this procedure. Some of the experimental examples are as follows

This section provides experimental examples to illustrate the procedure of assessing the performance of a PID control loop using the proposed IMC-IAE-based index. 18th IFAC World Congress (IFAC'11) Milano (Italy) August 28 - September 2, 20117489 In the experiments, the process is a water tank system, whose crosssectional area is about $320 \mathrm{~cm} 2$. The water level of the tank is selected as the process variable (PV), with the range $[0,100]$. The opening of the outlet valve is fixed, while the input valve is driven by a frequency convertor to control the inlet flow, i.e., the frequency of the converter is the manipulated variable. The PID controller is in the non-interactive form . The sampling period is $0.5 \mathrm{sec}$. Two experiments are performed for two different sets of PID controller parameters. In both experiments, the setpoint has initially been staying at the value 20 for a sufficient long time for the PV to initiate at the steady state. The setpoint experiences a ramp change as $\mathrm{r}(\mathrm{t})=20$ $+0.8 \mathrm{t}, 06 \mathrm{t}<25,40,256 \mathrm{t}<250$. (17) 050100150200250300152025303540455055 Time/s Water level of tank Closed loop ramp response Reference $\mathrm{Kp}=1.2, \mathrm{Ti}=10, \mathrm{Td}=0 \mathrm{Kp}=2.025, \mathrm{Ti}=233.94, \mathrm{Td}=0 \mathrm{Fig}$. 4. The ramp setpoint (dashed), the measured PV (solid) for $\mathrm{Kp}=1.2, \mathrm{Ti}=10$ and $\mathrm{Td}=0$, and the measured PV (dasheddotted) for $\mathrm{Kp}=2.0249, \mathrm{Ti}=233.94$ and $\mathrm{Td}=0$. In the first experiment, the PID controller parameters are $\mathrm{Kp}=$ 1.2, $\mathrm{Ti}=10$ and $\mathrm{Td}=0$, and the corresponding ramp response is shown in Fig. 4 (solid line). Based on this closed-loop ramp response, an FOPDT model for the open-loop process is estimated, $\mathrm{P}^{\wedge}(\mathrm{s})=5.7256211 .28 \mathrm{~s}+1$ e $-1.2143 \mathrm{~s}$. (18) The current closed-loop time constant is estimated by fitting another FOPDT model for the closed-loop system, $\tau \mathrm{c}=8.8196$. Thus, the lower bound of the IAE is IAE0 $=200.6780$. As the actual IAE of the response is 742.3969 , the IMC-IAE-based index is $\eta \mathrm{IAE}, \mathrm{y}(\mathrm{t})=0.2703$. To avoid an incorrect estimate of $\eta \mathrm{IAE}$ due to the noise, a noise free closed-loop response $\mathrm{y}^{\wedge}(\mathrm{t})$ is obtained as described in Section 3, based on $\mathrm{P}^{\wedge}(\mathrm{s})$ in (18) subject to the same setpoint $r(t)$ in (17); the resulted IAE (based on $y^{\wedge}(t)$ and $r(t)$ ) is 681.1547 and the IMCIAE-based index is $\eta \mathrm{IAE}, \mathrm{y}^{\wedge}(\mathrm{t})=0.2946$. The two indices are close to each other, saying that the current controlloop performance has a quite large space for improvement. All these results are summarized in Table 3 for clarity. In the second experiment, a different set of PID controller parameters is adopted, $\mathrm{Kp}=2.0249, \mathrm{Ti}=$ 233.94 and $\mathrm{Td}=0$.

\begin{tabular}{|l|l|l|}
\hline & 1st Experiment & 2nd Experiment \\
\hline$\tau_{c}$ & 8.8196 & 16.6068 \\
\hline$\theta$ & 1.2143 & 1.1122 \\
\hline $\mathrm{IAE}_{0}$ & 200.6780 & 354.3800 \\
\hline $\mathrm{IAE}_{y(t)}$ & 742.3969 & 446.9704 \\
\hline $\mathrm{IAE}_{\hat{\mathrm{y}}(t)}$ & 681.1547 & 364.5743 \\
\hline$\eta_{I A E_{, y(t)}}$ & 0.2703 & 0.7928 \\
\hline$\eta_{I A E_{\hat{y}(t)}}$ & 0.2946 & 0.9720 \\
\hline
\end{tabular}


Table 2. Experimental examples

The resulted ramp response experiences little overshooting, (dashed-dotted line). Based on this closed-loop ramp response, an FOPDT model for the open-loop process is estimated, $\mathrm{P}^{\wedge}(\mathrm{s})=6.6469241 .3700 \mathrm{~s}+1 \mathrm{e}-1.1122 \mathrm{~s}$. $r(t)= \begin{cases}20+0.8 t, & 0 \leqslant t<25, \\ 40 & 25 \leqslant t<250 .\end{cases}$

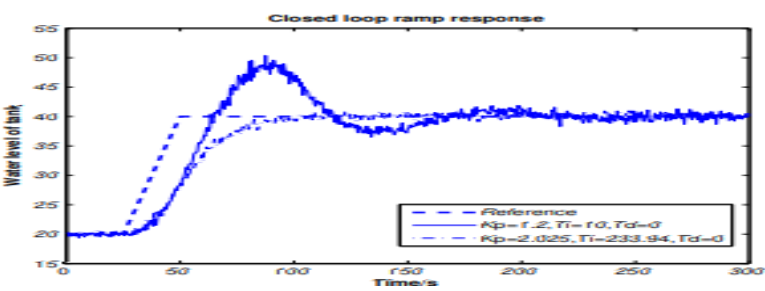

Fig 3.5.1 Closed Loop ramp response

From the Table 4.1 Experimental examples, Analogously to the first experiment, the performance assessment results are obtained. Both $\eta \mathrm{IAE}, \mathrm{y}(\mathrm{t})$ and $\eta \mathrm{IAE}, \mathrm{y}^{\wedge}(\mathrm{t})$ say that the control-loop performance is excellent and is very close to the expected one by using the IMC-tuning rule. In fact, from Fig 4.1 Closed Loop ramp response , the IMC tuning rule is used based on the model in (19), the resulted controller parameters are $\mathrm{Kp}=2.0494, \mathrm{Ti}=$ 241.37 and $\mathrm{Td}=0$, which are almost the same as the PID controller parameters used in the current experiment.

\section{CONCLUSION}

In the proposed method, This paper established the lower bound of the IAE for PID controllers tuned by following the IMC principle, from closed-loop responses subject to step, ramp or other types of setpoint changes. Based on the lower bound, an IMC-IAE-based index was proposed in (14) to assess the performance of PID control loops. Numerical examples validated the obtained lower bound as the performance benchmark. Experimental examples and an industrial case study illustrated the effectiveness of the IMC-IAE-based index.The IMC and IMC-based PID controller can successfully achieve any industrial process because it is present in the plant uncertainty parameters sufficiently strong. IMC-based PID controller algorithm is robust and simple processing model uncertainty, therefore, IMC-PID tuning method seems to be a useful tradeoff between performance closed-loop systems, we achieved robust build inaccurate single-mode tuning parameters. It also provides a good solution in the process of a significant time delay is actually working in the real-time situation. IMC has to compensate the model uncertainty and disturbance open-loop control does not have the ability to attach advantage.

\section{REFERENCES}

1. D.Prasanth Sai (2011). Performance Assessment of PID Control Loops based on IMC Tuning Rule. IFAC Proceedings Volumes, 44(1), 7486-7491. doi:10.3182/20110828-6-it-1002.01285.

2. U. SaburaBanu et al. (Eds.): SEMCCO 2010, LNCS 6466, pp. 648-655, 2010. (C Springer-Verlag Berlin Heidelberg 2010.

3. International Journal of Applied Engineering Research ISSN 0973-4562 Volume 13, Number 22 (2018) pp. 15922-15925.

4. 18th World Congress The International Federation of Automatic Control Milano (Italy) August 28 September 2, $2011,978-3-902661-93-7 / 11 / \$ 20.00$ C 2011 IFAC.

5. J.G. Ziegler, -Automatic Tuning of Commercial PID Controller for single loop \& multi loop applications, IEEE Control systems magazine.Pp.37-44, Feb. 24-25, 2006.

6. Maher Algreer, Matthew Armstrong, and Damian Giaouris,-Adaptive PD+I Controlof a Switch-Mode DC - DC PowerConverter Using a Recursive FIR Predictor, IEEE Trans onIndus. Applications, VOL. 47, NO. 5, 2011, 2135-2145.

7. Te-Jen Su, et al., -Fuzzy PID Controller Design using Synchronous Bacterial Foraging Optimization pp.693-43,2008.

8. Zhou Ying, -A Nonlinear PID Controller based on an Adaptive Genetic Algorithm,\| Second InternationalNetworks and Intelligent Systems, pp. 98-102, 2009.

9. AhmedRubaai,FuzzyNeural Network Controller for Brushless Drives, IEEETRANSACTIONS ON INDUSTRY APPLICATIONS, 47(6):(2011) 2391-2401.

10. Muguo Li, Da Liu, A Novel Adaptive Self-turned PID controller based on Recurrent-Wavelet-NeuralNetwork for PMSM Speed Servo Drive System, Advanced in Control Engineering and Information Science, 15 (2011) 282- 287.

11. YiLuo, -Optimization of PID Controller Parameters Based on an Improved Artificial Fish Swarm Algorithm, - ThirdInternational Workshop on Advanced Computational Intelligence, August 25-27, 2010-Suzhou, Jiangsu, China. 
12. Sönke Thomsen, PI Control, PI-Based State Space Control, and Model-Based Predictive Control for Drive Systems with Elastically Coupled Loads - A Comparative Study, IEEE Transactions on Industrial Electronics, 58(8): (2011) 3647-3658.

13. Chun-Fei Hsu, FPGA-based adaptive PID control of a DC motor driver via sliding-mode approach, Expert Systems with Applications, 38 (2011), 11866 - 11872.

14. Chen, D. Seborg, D. E. (2002). PI/PID controller design based on direct synthesis and disturbance rejection. Ind. Eng. Chem. Res., 41, pp. 4807-4822.

15. Sendjaja, A.Y. \&Kariwala, V. 'Achievable PID performance using sums of squares programming', Journal of Process Control, 19, 1061-1065, 2009.

16. Skogestad, S. 'Simple analytic rules for model reduction and PID controller tuning', Journal of Process Control, 13, 291-309, 2002.

17. Svante, B., \&Ljung, L. 'A survey and comparison of time-delay estimation methods in linear systems', Proc. of the 42nd IEEE Conf. Decision and Control, 2502- 2507, 2003.

18. Swanda, A.P. \&Seborg, D.E. 'Controller performance assessment based on set-point data', American Control Conference, 6, 3863-3867, 1999.

19. Tan, W., Marquez, H.J. \& Chen, T. 'Performance assessment of PID controllers', Control and Intelligent Systems, 32, 158-166, 2004.

20. Veronesi, M. \&Visioli, A. 'Performance assessment and retuning of PID controllers', Ind. Eng. Chem. Res., 48, 2616- [13]. Rivera D.E., M. Morai and S. Skogestad, (1986) "Internal Model Control. 4. PID Controller Design", Ind. Eng. Chem. Process Des. Dev.25, 252.

21. Rivera E Daniel, Skogestad, S, Internal Model Control-PID Controller Design. Chemical Engineering American Institute of technology.

22. Ogunnaike, B.A. and W.H. Ray. (1994) Process Dynamics, Modeling and Control, Oxford University Press, New York. Grimble, M.J. 'Restricted structure controller tuning and assessment', IEE Proceedings on Control Theory and Applications, 149, 8-16, 2002.

23. Hagglund, T. 'Industrial implementation of on-line performance monitoring tools', Control Engineering Practice, 13, 1383-1390, 2005.

24. Harris, T.J. 'Assessment of closed loop performance', Canadian Journal of Chemical Engineering, 67, 856- 861, 1989.

25. Vengatesan, K., Kumar, A., Naik, R., \& Verma, D. (2018). Anomaly based novel intrusion detection system for network traffic reduction. In 2018 2nd International Conference on I-SMAC (IoT in Social, Mobile, Analytics and Cloud)(I-SMAC) I-SMAC (IoT in Social, Mobile, Analytics and Cloud)(ISMAC), 2018 2nd International Conference on (pp. 688-690).

26. Huang, H.P. \&Jeng, J.C. 'Monitoring and assessment of control performance for single loop systems', Ind. Eng. Chem. Res., 41, 1297-1309, 2002.

27. Jelali, M. 'An overview of control performance assessment technology and industrial applications', Control Engineering Practise, 14, 441-466, 2006.

28. Kumar, A., Vengatesan, K., Rajesh, R., Parthibhan, M., \& Singhal, A. (2018). Review of Gene Subset Selection using Modified K-Nearest Neighbor Clustering Algorithm. In 2018 International Conference on Smart Systems and Inventive Technology (ICSSIT) (pp. 570-574) 